Pacific Journal of Mathematics

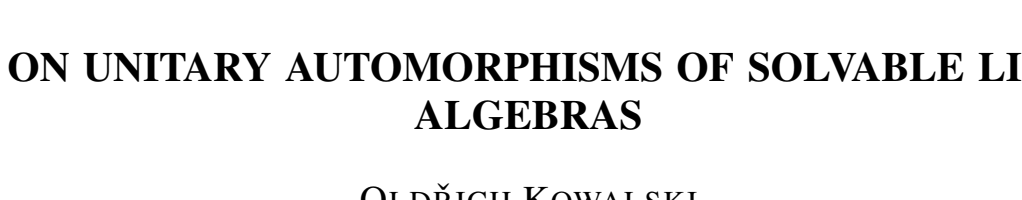




\title{
ON UNITARY AUTOMORPHISMS OF SOLVABLE LIE ALGEBRAS
}

\author{
OLDRICH KOWALSKI
}

\begin{abstract}
Let $V$ be a finite dimensional vector space over real numbers. An automorphism $A$ of $V$ is called unitary if it is semisimple and all its eigenvalues are complex units. Particularly, all periodic automorphisms, i.e., such that $T^{k}=$ identity for some integer $k$, are unitary. The aim of this paper is to prove the following Theorem. Let $g$ be an $n$-dimensional real Lie algebra admitting a unitary automorphism without nonzero fixed vectors. Then $g$ admits a periodic automorphism without nonzero fixed vectors and of order $k$, where $k \leq 5^{n / 4}$ for $n$ even, and $k \leqq 2.5^{(n-1) / 4}$ for $n$ odd.
\end{abstract}

The proof is based upon the detailed study of possible eigenvalues of admissible automorphisms of $\mathfrak{g}$. Yet our method is purely combinatorial-we do not make use of the Jacobi identities in $g$. Thus, the same method can be applied to nonassociative algebras or, more generally, to various tensor structures on vector spaces. As concerns applications to the differential geometry (generalized symmetric Riemannian spaces), see note at the end of this paper.

\section{Comments:}

(a) Obviously, an automorphism $A$ of $V$ is unitary if and only if $V$ admits a scalar product $\langle$,$\rangle such that \langle T u, T v\rangle=\langle u, v\rangle$ for all $u, v \in V$.

(b) It is well-known ([1], [3]) that a finite dimensional Lie algebra admitting an automorphism without nonzero fixed vectors is solvable. Thus our theorem is essentially a result on solvable Lie algebras.

(c) For the validity of our theorem, it is not necessary to assume that the initial automorphism is semisimple. For, let $A$ be an automorphism of $g$ and $A=S \cdot U$ the Jordan decomposition into the semisimple and the unipotent part. Then $S$ is an automorphism of $g$ possessing the same eigenvalues as $A$. Particularly, if $A$ is fixed-point free then so is $S$, and if all eigenvalues of $A$ are complex units then $S$ is unitary.

(d) The fact that $g$ admits a fixed-point free automorphism of finite order can be proved directly as follows (cf. also [2]): Let $A$ be the given unitary, fixed-point free automorphism of $\mathfrak{g}$; then $A$ can be represented by a diagonal matrix $\left(\theta_{1}, \cdots, \theta_{n}\right)$ belonging to 
the torus $T^{n}$. Denote by $G$ the closed subgroup of $T^{n}$ generated by the powers of $A$. Then $G$ is a product of a torus and a finite group. Hence the elements of finite order of $G$ are dense and the result follows.

Thus the principal result of our theorem is not the existence part but the given inequalities.

1. The space of eigenvalues-multiplicative theory. Let $\mathscr{B}^{n}$ denote the set of all $n$-tuples $\left(\theta_{1}, \cdots, \theta_{n}\right)$ of complex units such that $\theta_{i} \neq 1$ for $i=1, \cdots, n$. (Thus, as a submanifold of $C^{n}, \mathscr{B}^{n}$ is diffeomorphic to an open unit cube of $R^{n}$ ). A characteristic variety of $\mathscr{B}^{n}$ is a hypersurface $\mathscr{y} \subset \mathscr{B}^{n}$ defined by any of the following relations:

$$
\begin{array}{ll}
\theta_{i} \cdot \theta_{j}=\theta_{k} & (i \neq j \neq k ; i, j, k=1, \cdots, n) \\
\theta_{i} \cdot \theta_{j}=1 & (i, j=1, \cdots, n) \\
\theta_{i}=\theta_{j} & (i \neq j) .
\end{array}
$$

For a permulation $\pi \in \Sigma_{n}$ of the indices $1, \cdots, n$ and for a subset $\mathscr{M} \subset \mathscr{B}^{n}$ we put

$$
I_{\pi}(\mathscr{C l})=\left\{\left(\theta_{\pi(1)} \cdots, \theta_{\pi(n)}\right) /\left(\theta_{1}, \cdots, \theta_{n}\right) \in \mathscr{C}\right\} .
$$

If $\mathscr{V}$ is a characteristic variety then so is $I_{\pi}(\mathscr{V})$ for each $\pi \in \Sigma_{n}$. Finally, put

$$
\Sigma(\mathscr{C})=\bigcup_{\pi \in \Sigma_{n}} I_{\pi}(\mathscr{C}) \text { for all } \mathscr{C} \subset \mathscr{B}^{n} .
$$

By a $\theta$-variety of $\mathscr{B}^{n}$ we mean a nonempty set of the form $\Sigma\left(\mathscr{V}_{1} \cap \cdots \cap \mathscr{V}_{p}\right)$, where $\mathscr{V}_{1}, \cdots, \mathscr{V}_{p}$ are characteristic varieties. Obviously, we have only finite number of $\theta$-varieties in $\mathscr{B}^{n}$; they form a partially ordered set with respect to the inclusion map. Let $\mathscr{C}^{n} \subset \mathscr{B}^{n}$ be the union of all characteristic varieties of $\mathscr{B}^{n}$. For $\left(\theta_{i}\right) \in \mathscr{C}^{n}$ we shall denote by $\mathscr{W}\left(\theta_{i}\right)$ the intersection of all characteristic varieties containing $\left(\theta_{i}\right)$. Finally, we put $\mathscr{W}^{*}\left(\theta_{i}\right)=$ $\Sigma\left(\mathscr{W}\left(\theta_{i}\right)\right)$. Then $\mathscr{W}^{*}\left(\theta_{i}\right)$ is the minimum $\theta$-variety containing $\left(\theta_{i}\right)$. It is easy to see that, for each $\theta$-variety $\mathscr{W}$ of $\mathscr{B}^{n}$, there is $\left(\theta_{i}\right) \epsilon$ $\mathscr{W}$ such that $\mathscr{W}=\mathscr{W}^{*}\left(\theta_{i}\right)$.

Now, let $\mathscr{A}^{n}$ denote the subset of all elements $\left(\theta_{i}\right) \in \mathscr{C}^{n}$ with the following property: if $\theta$ is among $\theta_{1}, \cdots, \theta_{n}$ with the multiplicity $m$ then so is its conjugate $\bar{\theta}$. Equivalently: $\left(\theta_{i}\right) \in \mathscr{C}^{n}$ belongs to $\mathscr{A}^{n}$ if and only if there is a permutation $\rho \in \Sigma_{n}$ such that $\rho^{2}=i d$, and $\bar{\theta}_{i}=\theta_{\rho(i)}$ for $i=1, \cdots, n$. Obviously, if $S$ is a real unitary automorphism without nonzero fixed vectors of a real vector space $V^{n}$, then the system of eigenvalues $\left(\theta_{i}\right)$ of $S$ belongs to $\mathscr{A}^{n}$. 
For $\left(\theta_{i}\right) \in \mathscr{A}^{n}$ we always have $\mathscr{W}^{*}\left(\theta_{i}\right) \subset \mathscr{A}^{n}$. In fact, because $\mathscr{W}^{*}\left(\theta_{i}\right)=\Sigma\left(\mathscr{W}\left(\theta_{i}\right)\right)$, and $\Sigma\left(\mathscr{A}^{n}\right)=\mathscr{A}^{n}$, it is sufficient to prove $\mathscr{W}\left(\theta_{i}\right) \subset \mathscr{A}^{n}$. Suppose $\left(\theta_{i}\right) \in \mathscr{A}^{n}$, and let $\rho \in \Sigma_{n}$ be a permutation such that $\rho^{2}=i d$, and $\bar{\theta}_{i}=\theta_{\rho(i)}$ for $i=1, \cdots, n$. Then $\theta_{i} \theta_{\rho(i)}=1$ for $i=1, \cdots, n$. In other words, $\mathscr{W}\left(\theta_{i}\right) \subset \mathscr{W}_{1} \cap \cdots \cap \mathscr{W}_{n}$ where $\mathscr{W}_{i}$ denotes the characteristic variety of $\mathscr{B}^{n}$ given by the relation $\theta_{i} \theta_{\rho(i)}=1$. For $\left(\theta_{i}^{\prime}\right) \in \mathscr{W}\left(\theta_{i}\right)$ we get $\theta_{i}^{\prime} \theta_{\rho(i)}^{\prime}=1$ for $i=1, \cdots, n$, i.e., $\bar{\theta}_{i}^{\prime}=\theta_{\rho(i)}^{\prime}$ for each $i$. Hence $\left(\theta_{i}^{\prime}\right) \in \mathscr{A}^{n}$.

As a consequence, we can see that each minimal $\theta$-variety $\mathscr{Y} \subset \mathscr{B}^{n}$ either belongs to $\mathscr{A}^{n}$ or to the complement $\mathscr{B}^{n} \backslash \mathscr{A}^{n}$.

Proposition 1. Let $S$ be a unitary automorphism without nonzero fixed vectors of a real Lie algebra $\mathfrak{g}(\operatorname{dim} \mathfrak{g}=n)$. Let $\left(\theta_{i}\right) \in$ $\mathscr{A}^{n}$ be the system of eigenvalues of $S$. Then for each $n$-tuple $\left(\theta_{i}^{\prime}\right) \in$ $\mathscr{W}^{*}\left(\theta_{i}\right)$ there is a unitary automorphism $S^{\prime}$ of $\mathfrak{g}$ having $\left(\theta_{i}^{\prime}\right)$ as its system of eigenvalues.

Proof. Suppose $\left(\theta_{i}^{\prime}\right) \in \mathscr{W}^{*}\left(\theta_{i}\right)$. Then there is a permutation $\pi \epsilon$ $\Sigma_{n}$ such that $I_{\pi}\left(\theta_{i}^{\prime}\right) \in \mathscr{W}\left(\theta_{i}\right)$. Thus we can re-numerate the eigenvalues $\theta_{1}^{\prime}, \cdots, \theta_{n}^{\prime}$ in such a way that $\left(\theta_{i}^{\prime}\right) \in \mathscr{W}\left(\theta_{i}\right)$.

Let $U_{1}, \cdots, U_{n} \in \mathfrak{g}^{c}$ be a basis of (complex) eigenvectors corresponding to the eigenvalues $\theta_{1}, \cdots, \theta_{n}$ respectively. Moreover, we can suppose that a permutation $\rho \in \Sigma_{n}$ exists such that $\rho^{2}=i d, \bar{\theta}_{i}=$ $\theta_{\rho(i)}, \bar{U}_{i}=U_{\rho(i)}$ for $i=1, \cdots, n$. Now, let us define a linear transformation $S^{\prime}$ of $\mathrm{g}^{c}$ by the relations $S^{\prime} U_{i}=\theta_{i}^{\prime} U_{i}, i=1, \cdots, n$. We have to show that $S^{\prime}$ induces a (real) automorphism of $\mathrm{g}$.

Firstly, $S^{\prime}$ induces a real linear transformation of g. In fact, we have $\bar{\theta}_{i}^{\prime}=\theta_{\rho(i)}^{\prime}$ for each $i$, and hence $S^{\prime} U_{i}=\theta_{i}^{\prime} U_{i}$ implies $S^{\prime} \bar{U}_{i}=$ $S^{\prime} U_{\rho(i)}=\theta_{\rho(i)}^{\prime} U_{\rho(i)}=\bar{\theta}_{i}^{\prime} \bar{U}_{i}$. Further, put $\left[U_{i}, U_{j}\right]=\sum_{k=1}^{n} c_{i j}^{k} U_{k}$. Then because $S$ is an automorphism, we have $S\left(\left[U_{i}, U_{j}\right]\right)=\left[S U_{i}, S U_{j}\right]=$ $\theta_{i} \theta_{j}\left[U_{i}, U_{j}\right]$. Thus $\left[U_{i}, U_{j}\right] \neq 0$ implies that $\theta_{i} \theta_{j}$ is an eigenvalue of $S$ and $c_{i j}^{k} \neq 0$ can occur only in case that $\theta_{k}=\theta_{i} \theta_{j}$. Now, $S^{\prime}\left(\left[U_{i}, U_{j}\right]\right)=\sum_{k=1}^{n} c_{i j}^{k} \theta_{k}^{\prime} U_{k}$, where $c_{i j}^{k} \neq 0$ only if $\theta_{k}^{\prime}=\theta_{i}^{\prime} \theta_{j}^{\prime}$. Hence $S^{\prime}\left(\left[U_{i}, U_{j}\right]\right)=\theta_{i}^{\prime} \theta_{j}^{\prime}\left[U_{i}, U_{j}\right]=\left[S^{\prime} U_{i}, S^{\prime} U_{j}\right]$. This completes the proof.

Now, consider the partially ordered set of all $\theta$-varieties of $\mathscr{B}^{n}$, and let $\mathscr{D}^{n}$ denote the union of all minimal $\theta$-varieties (with respect to the inclusion map). We obtain the following consequence of Proposition 1:

Proposition 2. Let $\mathfrak{g}$ be an n-dimensional real Lie algebra admitting a unitary automorphism without nonzero fixed vectors. Then $\mathfrak{g}$ admits a unitary automorphism $S$ such that its system of eigenvalues belongs to $\mathscr{A}^{n} \cap \mathscr{D}^{n}$. 
For our further purposes, we have to reveal the structure of the variety $\mathscr{D}^{n}$. In the next paragraphs we shall show that $\mathscr{D}^{n}$ is a finite set. There is a certain difficulty connected with the proof-it is caused by the fact that the manifold $\mathscr{B}^{n}$ is noncompact.

2. The space of eigenvalues-additive theory. We shall start this section with a geometric result called "Basic Lemma".

Let $\Lambda_{n}$ be a finite set of linear subspaces of the cartesian space $R^{n}\left[x^{1}, \cdots, x^{n}\right]$ with the following properties:

(a) For $K, L \in \Lambda_{n}$ we have $K \cap L \in \Lambda_{n}$.

(b) $\Lambda_{n}$ contains all hyperplanes given by the equations of the form $x^{i}-x^{j}=0$, or $x^{i}+x^{j}=1$, where $1 \leqq i<j \leqq n$.

Let $\left(I^{n}\right)^{0}$ denote the open unit cube,

$$
\left(I^{n}\right)^{0}=\left\{\left[x^{1}, \cdots, x^{n}\right] \in R^{n} / 0<x^{i}<1, i=1, \cdots, n\right\} .
$$

$\mathscr{L}_{n} \subset \Lambda_{n}$ will denote the subset of all 0 -dimensional subspaces; they will be called the lattice points of $\Lambda_{n}$.

BASIC LEMMA. Each linear subspace $K \in \Lambda_{n}$ of dimension $k>0$ such that $K \cap\left(I^{n}\right)^{0} \neq \varnothing$ contains a proper subspace $L \in A_{n}$ of dimension $l<k$ such that $L \cap\left(I^{n}\right)^{0} \neq \varnothing$. Particularly, if $K \in \Lambda_{n}, K \cap$ $\left(I^{n}\right)^{0} \neq \varnothing$, then $K \cap\left(I^{n}\right)^{0}$ contains a lattice point $p \in \mathscr{L}_{n}$.

We shall send two more lemmas beforehand.

Lemma 1. (Generalized Pasch's axiom). Let $\Delta^{(r)}$ be an r-dimensional simplex in the euclidean space $E^{r}$, and let a linear subspace $K \subset E^{r}, \operatorname{dim} K>0$, intersect the interior of $\Delta^{(r)}$. Then $K$ intersects the interior of $a(r-1)$-dimensional face $\Delta_{i}^{(r-1)}$ and the interior of an l-dimensional face $\Delta_{j}^{(l)}(l \leqq r-1)$ such that $\Delta_{j}^{(l)} \nsubseteq \Delta_{i}^{(r-1)}$.

Proof is left to the reader.

Let $I^{n}(n \geqq 2)$ denote the closed $n$-dimensional unit cube

$$
I^{n}=\left\{\left[x^{1}, \cdots, x^{n}\right] \in R^{n} / 0 \leqq x^{i} \leqq 1, i=1, \cdots, n\right\} .
$$

LEMMA 2. $I^{n}$ admits a triangulation into $2^{n-1} \cdot n !$ simplexes $\Delta_{i}^{(n)}$ of dimension $n$ such that:

(a) Each $(n-1)$-dimensional face of each simplex $\Delta_{i}^{(n)}$ is contained in one of the following hyperplanes:

(2) $x^{i}=0$, or $x^{i}=1$, or $x^{i}-x^{j}=0(i \neq j)$, or $x^{i}+x^{j}=1(i \neq j)$.

(b) Exactly one $(n-1)$-dimensional face of each simplex $\Delta_{i}^{(n)}$ 
belongs to the boundary $\partial I^{n}$ of $I^{n}$.

(c) The center of the cube $I^{n}$ is the common vertex of all simplexes $\Delta_{i}^{(n)}$.

Proof. For $n=2$, the wanted triangulation of $I^{2}$ is given by means of 6 lines $x^{1}=0, x^{2}=0, x^{1}=1, x^{2}=1, x^{1}-x^{2}=0, x^{1}+x^{2}=1$. Suppose Lemma 2 to be true for some $n$, and consider the cube $I^{n+1}$. Let $T$ be a triangulation of $I^{n}$ satisfying the conditions of Lemma 2 , and let $f_{i, \alpha}: I^{n} \rightarrow I^{n+1}(i=1, \cdots, n+1 ; \alpha=0,1)$ denote the map

$$
f_{i, \alpha}\left(\left[x^{1}, \cdots, x^{n}\right]\right)=\left[x^{1}, \cdots, x^{i-1}, \alpha, x^{i+1}, \cdots, x^{n}\right] .
$$

Then we get a triangulation $f_{i, \alpha}(T)$ on each face $f_{i, \alpha}\left(I^{n}\right)$ of $I^{n+1}$, and thus a triangulation of the boundary $\partial\left(I^{n+1}\right)$. Now, we define a triangulation $T^{\prime}$ of $I^{n+1}$ in such a way that each $(n+1)$-dimensional simplex $\Delta_{i}^{(n+1)}$ of $T^{\prime}$ has the center $[1 / 2, \cdots, 1 / 2]$ of $I^{n+1}$ for a vertex and an $n$-dimensional simplex of the boundary triangulation for a face. It is obvious that $T^{\prime}$ consists of $2 n \cdot 2^{n-1} \cdot n !=$ $2^{n} \cdot(n+1)$ ! simplexes $\Delta_{i}^{(n+1)}$, and that the conditions $(\mathrm{b}),(\mathrm{c})$ are also satisfied. The verification of the condition (a) is left to the reader.

LEMma 3. Let $T$ be a triangulation of the cube $I^{n}$ satisfying the conditions of Lemma 2. Further, let $\Lambda_{n}$ be a set of linear subspaces of $R^{n}$ as in Basic Lemma. Then each k-dimensional simplex $\Delta_{j}^{(k)}(k \leqq n-1)$ of the triangulation $T$ is either contained in the boundary $\partial I^{n}$, or it is contained in a linear subspace $K \in \Lambda_{n}$ and has the point $[1 / 2, \cdots, 1 / 2]$ for a vertex.

Proof. Each $k$-dimensional simplex $\Delta_{i}^{(k)}$ of $T$ is the intersection of a certain number of $(n-1)$-dimensional simplexes of $T$. Thus the $k$-dimensional plane $L$ containing $\Delta_{i}^{(k)}$ is determined by a system of $n-k$ equations of the form (2). Now, if $\Delta_{i}^{(k)}$ is not contained in the boundary $\partial I^{n}$, it has the point $[1 / 2, \cdots, 1 / 2]$ for a vertex. Hence $L$ is given by a system of equations of the form $x^{i}-x^{j}=0$. $x^{l}+x^{m}=1$, and thus it belongs to $\Lambda_{n}$.

Proof of Basic Lemma. Let $T$ be the triangulation of $I^{n}$ constructed in Lemma 2. Let $K \in \Lambda_{n}$ be of dimension $k>0$ and such that $K \cap\left(I^{n}\right)^{0} \neq \varnothing$. Let $\Delta_{i}^{(r)} \in T$ be a simplex of the minimum dimension containing a point of $K \cap\left(I^{n}\right)^{0}$ in its interior. Let $L \supset$ $\Delta_{i}^{(r)}$ be the corresponding $r$-dimensional plane. According to Lemma 3 , we have $L \in \Lambda_{n}$, and $[1 / 2, \cdots, 1 / 2]$ is a vertex of $\Delta_{i}^{(r)}$.

Suppose now $K \subseteq L$. Then, according to Lemma 1 , the $k$ - 
dimensional plane $K$ intersects the interior of a face $\Delta_{u}^{(r-1)}$ and the interior of a face $\Delta_{v}^{(l)}$, where $l \leqq r-1$ and $\Delta_{v}^{(l)} \nsubseteq \Delta_{u}^{(r-1)}$. Then at least one of the simplexes $\Delta_{u}^{(r-1)}, \Delta_{v}^{(l)} \in T$ contains the vertex $[1 / 2, \cdots$, $1 / 2$ ] and thus it does not belong to the boundary $\partial I^{n}$. Moreover, it contains in its interior a point $q \in K \cap\left(I^{n}\right)^{0}-a$ contradiction to the minimality of $r$.

Hence $K \nsubseteq L$, and the subspace $P=K \cap L$ is a proper subspace of $K$. We have $P \in \Lambda_{n}$ and $P \cap\left(I^{n}\right)^{0} \neq \varnothing$, which completes the proof.

Proposition 3. The union $\mathscr{D}^{n}$ of all minimal $\theta$-varieties of $\mathscr{B}^{n}$ is a finite set. Moreover, each element $\left(\theta_{i}\right) \in \mathscr{D}^{n}$ is of finite order, i.e., $\left(\theta_{1}\right)^{k}=\left(\theta_{2}\right)^{k}=\cdots=\left(\theta_{n}\right)^{k}=1$ for some integer $k$.

Proof. Consider the diffeomorphism $f$ of the open unit cube $\left(I^{n}\right)^{0} \subset R^{n}$ onto $\mathscr{B}^{n}$ given as follows:

$$
f\left(\left[x^{1}, \cdots, x^{n}\right]\right)=\left(\exp \left(2 \pi \sqrt{-1} x^{1}\right), \cdots, \exp \left(2 \pi \sqrt{-1} x^{n}\right)\right) .
$$

Consider the set of all hyperplanes of $R^{n}$ which correspond to the following linear equations:

$$
\begin{array}{ll}
x^{i}+x^{j}-x^{k}=0, & (i \neq j \neq k \neq i ; i, j, k=1, \cdots, n) \\
x^{i}+x^{j}-x^{k}=1 ; & (i \neq j ; i, j=1, \cdots, n) \\
x^{i}+x^{j}=1, & (i=1, \cdots, n) . \\
x^{i}-x^{j}=0 ; &
\end{array}
$$

Let $\Lambda_{n}$ denote the set of all linear subspaces of $R^{n}$ which are intersections of finite number of hyperplanes given above. For each $L \in \Lambda_{n}$ we shall take into consideration all hyperplanes of the form (3) containing $L$. Thus, each subspace $L \in \Lambda_{n}$ is characterized by a unique (maximal) set $\mu(L)$ of linear nonhomogeneous equations of the form (3); the equations of this set may be linearly dependent. For each subspace $L$ we also consider the corresponding set $h(L)$ of linear homogeneous equations. Now, two subspaces $L, L^{\prime} \in \Lambda_{n}$ will be said to be conjugate if $h(L)=h\left(L^{\prime}\right)$.

For any permutation $\pi \in \Sigma_{n}$ consider the transformation

$$
I_{\pi}:\left[x^{1}, \cdots, x^{n}\right] \longmapsto\left[x^{\pi(1)}, \cdots, x^{\pi(n)}\right]
$$

of $R^{n}$, and for $L \subset R^{n}$ put $\Sigma(L)=\bigcup_{\pi \in \Sigma_{n}} I_{\pi}(L)$.

LEMma 4. If $\mathscr{W} \subset \mathscr{B}^{n}$ is an $\theta$-variety then $f^{-1}(\mathscr{W})=\Sigma\left(L_{1} \cup\right.$ $\left.\cdots \cup L_{r}\right) \cap\left(I^{n}\right)^{0}$, where $L_{1}, \cdots, L_{r} \in \Lambda_{n}$ is a complete set of mutually 
conjugate subspaces. Conversely, if $L_{1}, \cdots, L_{r} \in A_{n}$ is a complete set of mutually conjugate subspaces then $f\left[\Sigma\left(L_{1} \cup \cdots \cup L_{r}\right) \cap\left(I^{n}\right)^{0}\right]$ is either an empty set or a $\theta$-variety.

The proof is easy and it is left to the reader.

Lemma 5. If $\mathscr{W} \subset \mathscr{B}^{n}$ is a minimal $\theta$-variety then $f^{-1}(\mathscr{W})$ consists of lattice points of $\Lambda_{n}$.

Proof of the lemma. Let $\mathscr{W}$ be a $\theta$-variety and put $f^{-1}(\mathscr{W})=$ $\Sigma\left(L_{1} \cup \cdots \cup L_{r}\right) \cap\left(I^{n}\right)^{0}$. Suppose that $\operatorname{dim} L_{1}>0, L_{1} \cap\left(I^{n}\right)^{0} \neq \varnothing$. The set $\Lambda_{n}$ satisfies the conditions of Basic Lemma. Thus, there is a lattice point $p \in L_{1} \cap\left(I^{n}\right)^{0}$. Obviously, the set $L_{1} \cup \cdots \cup L_{r}$ contains all lattice points $p_{1}, \cdots, p_{s}$ which are conjugate to $p$, and $f\left(\Sigma\left\{p_{1}, \cdots\right.\right.$, $\left.\left.p_{s}\right\} \cap\left(I^{n}\right)^{0}\right) \subset \mathscr{W}$ is a $\theta$-variety. Hence Lemma 5 follows.

Proof of Proposition 3-continuation. Clearly, the points of the set $\mathscr{D}^{n}$ are in one-to-one correspondence with the lattice points of $\Lambda_{n}$ included in $\left(I^{n}\right)^{0}$. Now, all lattice points of $\Lambda_{n}$ have rational coordinates, and consequently, the points $\left(\theta_{i}\right)$ of $\mathscr{D}^{n}$ are elements of finite order.

3. Evaluation of an upper bound for the order.

Proposition 4. Let $k(n)$ denote the maximum order of an element of $\mathscr{D}^{n} \cap \mathscr{A}^{n}$. Then $k(n) \leqq 5^{n / 4}$ for $n$ even, and $k(n) \leqq$ $2.5^{(n-1) / 4}$ for $n$ odd.

The proof will be performed, after some preparations, at the end of this section.

Let $\left[a^{1}, \cdots, a^{n}\right] \in\left(I^{n}\right)^{0}$ be a lattice point of $\Lambda_{n}$. If $\left(\theta_{1}, \cdots, \theta_{n}\right)=$ $f\left(\left[a^{1}, \cdots, a^{n}\right]\right) \in \mathscr{A}^{n}$, then there is a permutation $\rho \in \Sigma_{n}$ such that $\rho^{2}=$ identity, and $\bar{\theta}_{i}=\theta_{\rho(i)}$, i.e., $a^{i}+a^{\rho(i)}=1$ for $i=1, \cdots, n$. Suppose $\rho(i) \neq i$ for $i=1, \cdots, 2 r$ and $\rho(j)=j$ for $j=2 r+1, \cdots, n$. We can also achieve by a re-numeration that $0<a^{i}<1 / 2$ for $i=$ $1, \cdots, r$ and $\rho(i)=r+i$ for $i=1, \cdots, r$. Naturally, we have $a^{2 r+1}=\cdots=a^{n}=1 / 2$. Put $b^{i}=a^{i}$ for $i=1, \cdots, r$, and $b^{j}=a^{r+j}$ for $j=r+1, \cdots, n-r$. Now, the lattice point $\left[a^{1}, \cdots, a^{n}\right]$ can be calculated from the values of $b^{1}, \cdots, b^{n-r}$. On the other hand, the numbers $a^{1}, \cdots, a^{n}$ are uniquely determined by a set $\mu\left(\left(a^{i}\right)\right)$ of linear equations of the form (3). Thus the corresponding values $b^{1}, \cdots, b^{r}, b^{r+1}, \cdots, b^{n-r}$ are uniquely determined by a set of equations of the form 


$$
\begin{aligned}
& y^{i}+y^{j}-y^{k}=0, \quad(i \neq j \neq k \neq i ; i, j, k=1, \cdots, n-r) \\
& y^{i}+y^{j}+y^{k}=1 ; \quad \\
& 2 y^{i}-y^{k}=0,2 y^{i}+y^{k}=1, y^{i}-y^{k}=0 \quad(i \neq k, i, k=1, \cdots, n-r) \\
& 2 y^{i}=1 \quad(i=r+1, \cdots, n-r) .
\end{aligned}
$$

It means that we can select a system of $n-r$ independent equations of the form (4) and then calculate $b^{1}, \cdots, b^{n-r}$ using the Cramer's rule.

Let $\Delta$ be the matrix of the left hand side of this system, and let $|\Delta|$ denote the absolute value of $\operatorname{det} \Delta$. Obviously, if we can find an upper bound for $|\Delta|$, we get hence an upper bound for the order of $\left(\theta_{i}\right)$. For this reason, we shall now investigate the matrices of the above type.

In the following, define the weight of a row (or column) of a matrix $\Delta$ to be the sum of the absolute values of all elements of this row (or column). For the sake of brevity, a matrix $\Delta$ will be said to be of type $\delta$ if its elements $a_{i}^{j}$ are integers satisfying $\left|a_{i}^{3}\right| \leqq 2$ and the weight of each row is $\leqq 3$. Clearly, a submatrix of a matrix of type $\delta$ is also of type $\delta$.

LEMma 6. Let $A_{n}(n \geqq 3)$ be a square matrix of type $\delta$ such that $\left|a_{i}^{j}\right| \leqq 1$ for $i, j=1, \cdots, n$. Let $w\left(\Delta_{n}\right)$ denote the number of all places in $\Delta_{n}$ occupied by the elements \pm 1 and $t\left(\Delta_{n}\right)=3 n-w\left(\Delta_{n}\right)$ Then

$$
\left|\Delta_{n}\right| \leqq 5^{n / 2}\left(\frac{\sqrt{5}}{3}\right)^{k}, \text { where } k=\min \left(2, t\left(\Delta_{n}\right)\right)
$$

Proof. Let us remark first that $t\left(A_{n}\right) \geqq 0$ for each $\Delta_{n}$. We shall now proceed by induction. For $n=3$ we can verify formula (5) directly. Suppose (5) to be true for all $n \leqq m, m \geqq 3$, and choose a matrix $\Delta_{m+1}$.

(A) Let us have first $t\left(\Delta_{m+1}\right)=0$ or 1 . We can suppose that $\Delta_{m+1}$ has exactly 3 elements \pm 1 in the first row. Denote by $D_{m+1}$ the matrix consisting of the remaining $m$ rows. If some column of $D_{m+1}$ consists of zeros then either $\Delta_{m+1}=0$, or $\Delta_{m+1}$ has a column of weight one. In the last case $\left|\Delta_{m+1}\right|=\left|\Delta_{m}\right|$, where $\Delta_{m}$ is a submatrix of $D_{m+1}$. Here $t\left(\Delta_{m}\right)=t\left(\Delta_{m+1}\right)$, and the induction step follows.

Let now each column of $D_{m+1}$ contain at least one element \pm 1 . Then each submatrix $\Delta_{m}^{(i)}$ of $D_{m+1}$ satisfies $t\left(\Delta_{m}^{(i)}\right) \geqq t\left(\Delta_{m+1}\right)+1$. If we put $k_{i}=\min \left(2, t\left(\Delta_{m}^{(i)}\right)\right), k=\min \left(2, t\left(\Delta_{m+1}\right)\right)$, then $k_{i} \geqq k+1$ for each $i$. Now, $\left|\Delta_{m}^{(i)}\right| \leqq 5^{m / 2}(\sqrt{5} / 3)^{k_{i}}$ 'according to (5). Hence $\left|\Delta_{m+1}\right| \leqq$ $3 \cdot \max _{i}\left|\Delta_{m}^{(i)}\right| \leqq 3 \cdot 5^{m / 2}(\sqrt{5} / 3)^{k+1} \leqq 5^{m+1 / 2}(\sqrt{5} / 3)^{k}$.

(B) Let now $t\left(\Delta_{m+1}\right) \geqq 2$. We shall distinguish two subcases: 
(B1) One row of $\Delta_{m+1}$ has weight 1 ; then $\left|\Delta_{m+1}\right|=\left|\Delta_{m}\right|$, where $\Delta_{m}$ is a suitable submatrix. Now $\left|\Delta_{m}\right| \leqq 5^{m / 2}$ and hence $\left|\Delta_{m+1}\right| \leqq 5^{m / 2} \leqq$ $5^{m+1 / 2}(\sqrt{5} / 3)^{2}$.

(B2) One row of $\Delta_{m+1}$ is of weight 2. The matrix $D_{m+1}$ consisting of remaining $m$ rows contains $3(m+1)-t\left(\Delta_{m+1}\right)-2=3 m-$ $\left(t\left(\Delta_{m+1}\right)-1\right)$ elements \pm 1 . If some column of $D_{m+1}$ consists of zeros, then either $\Delta_{m+1}=0$, or $\left|\Delta_{m+1}\right|=\left|\Delta_{m}\right|$ where $t\left(\Delta_{m}\right) \geqq t\left(\Delta_{m+1}\right)-1 \geqq 1$. In the last case $\left|\Delta_{m+1}\right|=\left|\Delta_{m}\right| \leqq 5^{m / 2}(\sqrt{5} / 3) \leqq 5^{(m+1) / 2}(\sqrt{5} / 3)^{2}$. If each column of $D_{m+1}$ contains at least one \pm 1 , then for each submatrix $\Delta_{m}^{(i)}$ of $D_{m+1}$ we have $t\left(\Delta_{m}^{(i)}\right) \geqq t\left(\Delta_{m+1}\right) \geqq 2$. Hence $\left|\Delta_{m}^{(i)}\right| \leqq 5^{m / 2}(\sqrt{5} / 3)^{2}$, and $\left|\Delta_{m+1}\right| \leqq 2 . \max _{i}\left|\Delta_{m}^{(i)}\right| \leqq 2 \cdot 5^{m / 2}(\sqrt{5} / 3)^{2} \leqq 5^{m+1 / 2}(\sqrt{5} / 3)^{2}$.

Lemma 7. For an arbitrary matrix $\Delta_{n}$ of type $\delta$ we have

$$
\left|\Delta_{n}\right| \leqq 5^{n / 2}
$$

Proof. We can verify easily that $\left|\Delta_{1}\right| \leqq 2<5^{1 / 2},\left|\Delta_{2}\right| \leqq 5$, and thus the formula is true for $n=1,2$. Let (6) be true for all $n \leqq m$, and consider some $\Delta_{m+1}$. If $\Delta_{m+1}$ contains some row or column of weight $\leqq 2$, then we can write $\left|\Delta_{m+1}\right| \leqq 2 \cdot \max _{i}\left|\Delta_{m}^{(i)}\right| \leqq 2.5^{m / 2} \leqq 5^{m+1 / 2}$. (Here $A_{m}^{(i)}$ are the submatrices which are complementary to the elements of the given row or column.)

Suppose now that the weight of each column and each row is $\geqq 3$. (For the rows the weight is always 3.) If $\Delta_{m+1}$ does not contain elements \pm 2 , we have $\left|\Delta_{m+1}\right| \leqq 5^{m+1 / 2}$ according to Lemma 6. Thus, let $\Delta_{m+1}$ contain at least one element \pm 2 . Then interchanging the rows, and also the columns properly we can achieve that $a_{2}^{1}= \pm 2, a_{1}^{1} \neq 0, a_{1}^{2} \neq 0$. We shall consider 3 cases:

(a) $\left|a_{1}^{1}\right|=2,\left|a_{1}^{2}\right|=1$; then the submatrix $\Delta_{1}^{1}$ to $a_{1}^{1}$ has the first row of weight 1 , and the submatrix $\Delta_{1}^{2}$ to $a_{1}^{2}$ has at least one row of weight $\leqq 2$. Hence $\left|\Delta_{1}^{1}\right| \leqq 5^{m-1 / 2},\left|\Delta_{1}^{2}\right| \leqq 2 \cdot 5^{m-1 / 2},\left|\Delta_{m+1}\right| \leqq\left|a_{1}^{1}\right|\left|\Delta_{1}^{1}\right|+$ $\left|a_{1}^{2}\right|\left|\Delta_{1}^{2}\right| \leqq 4 \cdot 5^{m-1 / 2} \leqq 5^{m+1 / 2}$.

(b) $\left|a_{1}^{1}\right|=\left|a_{1}^{2}\right|=\left|a_{1}^{3}\right|=1$. Then the submatrix $\Delta_{1}^{1}$ has the first row of weight 1 , and each of the submatrices $\Delta_{1}^{2}, \Delta_{1}^{3}$ has at least one row of weight $\leqq 2$. Thus $\left|\Delta_{m+1}\right| \leqq \sum_{t}\left|a_{1}^{i}\right|\left|\Delta_{1}^{i}\right| \leqq(1+2+2) 5^{m-1 / 2} \leqq$ $5^{m+1 / 2}$.

(c) $\left|a_{1}^{1}\right|=1,\left|a_{1}^{2}\right|=2$. The conclusion $\left|\Delta_{m+1}\right| \leqq 5^{m+1 / 2}$ is quite similar to the case (b).

This completes the proof.

REMARK. For $n$ even, the estimate (6) is the best one.

Proof of Proposition 4. Consider a given $\left(\theta_{i}\right) \in \mathscr{D}^{n} \cap \mathscr{A}^{n}$ and 
the lattice point $\left[a^{1}, \cdots, a^{n}\right]=f^{-1}\left(\theta_{i}\right)$. Then we can find a compatible system of $n-r$ independent equations of the form (4) defining the corresponding values $b^{1}, \cdots, b^{r}, b^{r+1}, \cdots, b^{n-r}$. Without the loss of generality we can suppose that all equations of the form $2 y^{j}=1$ $(j=r+1, \cdots, n-r)$ form a part of this system. The matrix of the left hand side of the system take on the form

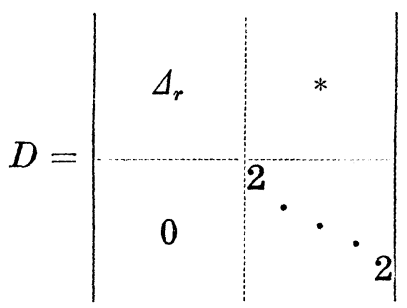

where $\Delta_{r}$ is a matrix of type $\delta$ with $r$ rows and columns. Solving the system by means of the Cramer's rule, we obtain for $i=1, \cdots$, $r:\left|y^{i}\right|=\left|D_{i}\right| /\left(2^{n-2 r} \cdot\left|\Delta_{r}\right|\right)$, where $D_{i}$ is the matrix obtained by replacing the $i$ th column of $D$ by a column consisting of elements 0 and \pm 1 . Clearly, if $n-2 r>0$, then $\left|D_{i}\right|=2^{n-2 r-1} \cdot n_{i}$, where $n_{i}$ is an integer. Hence $\left|b^{i}\right|=n_{i} /\left(2\left|\Delta_{r}\right|\right)(i=1, \cdots, r), b^{j}=1 / 2(j=r+1, \cdots$, $n-r)$. Thus, if $n-2 r>0$, we can see that $2\left|\Delta_{r}\right| \cdot b^{i}$ is an integer for $i=1, \cdots, n-r$, and also $2\left|\Delta_{r}\right| a^{j}$ is an integer for $j=1, \cdots, n$. If $n-2 r=0$, then $\left|\Delta_{r}\right| b^{i}$ is an integer for $i=1, \cdots, r$, and also $\left|\Delta_{r}\right| a^{j}$ is an integer for $j=1, \cdots, n$.

Now, if $n$ is odd, then $n-2 r>0$, and $\left(\theta_{i}\right)$ is of order $k \leqq$ $2\left|\Delta_{r}\right|$, where $\left|\Delta_{r}\right| \leqq 5^{r / 2} \leqq 5^{(n-1) / 4}$ according to Lemma 7 . If $n$ is even, then either $\left(\theta_{i}\right)$ is of order $k \leqq\left|\Delta_{r}\right|$, where $r=n / 2$, or of order $k \leqq 2\left|\Delta_{r}\right|$, where $r \leqq(n-2) / 2$. In both cases, $k \leqq 5^{n / 4}$. This completes the proof.

Now, our theorem follows from Proposition 2 and Proposition 4.

4. Note. In the paper [2] we have defined a generalized symmetric Riemannian space of order $k$. (Here, the usual globally symmetric Riemannian spaces are those of order 2.) We have also shown that all simply connected generalized symmetric Riemannian spaces are in one-to-one correspondence with certain tensor structures on vector spaces, admitting unitary automorphisms without nonzero fixed vectors. By the same method as we used above we can come to the following theorem:

Let $M$ be a simply connected generalized symmetric Riemannian space of dimension $n$ and of order $k$. Then $k \leqq 5^{n / 4}$ for $n$ even, and $k \leqq 2 \cdot 5^{(n-1) / 4}$ for $n$ odd. 


\section{REFERENCES}

1. A. Borel and G. D. Mostow, On semi-simple automorphisms of Lie algebras, Annals of Mathematics, 61 (1955), 389-405.

2. O. Kowalski, Riemannian manifolds with general symmetries, Math. Z., 136 (1974), 137-150.

3. D. J. Winter, On groups of automorphisms of Lie algebras, J. Algebra, 8 (1968), 131-142.

Received August 14, 1975 and in revised form December 21, 1978.

Charles University

SOKOLOVSKA 83

18600 Prague, Czechoslovakia 



\section{PACIFIC JOURNAL OF MATHEMATICS}

\section{EDITORS}

DoNALD BABBITT (Managing Editor)

University of California

Los Angeles, California 90024

HUGo Rossi

University of Utah

Salt Lake City, UT 84112

C. C. MOORE and ANDREW OGG

University of California

Berkeley, CA 94720
J. DUGUNDJI

Department of Mathematics University of Southern Californı Los Angeles, California 90007

R. Finn and J. Milgram Stanford University Stanford, California 94305

\section{ASSOCIATE EDITORS}

E. F. BECKENBACH

B. H. Neumann
F. WOLF

K. YOSHIDA

\section{SUPPORTING INSTITUTIONS}

UNIVERSITY OF BRITISH COLUMBIA CALIFORNIA INSTITUTE OF TECHNOLOGY UNIVERSITY OF CALIFORNIA MONTANA STATE UNIVERSITY UNIVERSITY OF NEVADA, RENO NEW MEXICO STATE UNIVERSITY OREGON STATE UNIVERSITY UNIVERSITY OF OREGON
UNIVERSITY OF SOUTHERN CALIFORNIA STANFORD UNIVERSITY UNIVERSITY OF HAWAII UNIVERSITY OF TOKYO UNIVERSITY OF UTAH WASHINGTON STATE UNIVERSITY UNIVERSITY OF WASHINGTON 


\section{Pacific Journal of Mathematics}

Vol. 82, No. $1 \quad$ January, 1979

Werner Bäni, Subspaces of positive definite inner product spaces of countable dimension ...................................... 1

Marilyn Breen, The dimension of the kernel of a planar set..............

Kenneth Alfred Byrd, Right self-injective rings whose essential right ideals

are two-sided

Patrick Cousot and Radhia Cousot, Constructive versions of Tarski's fixed

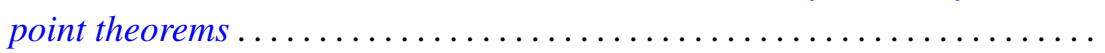

Ralph S. Freese, William A. Lampe and Walter Fuller Taylor, Congruence lattices of algebras of fixed similarity type. $I \ldots \ldots \ldots \ldots \ldots \ldots \ldots$

Cameron Gordon and Richard A. Litherland, On a theorem of Murasugi .....

Mauricio A. Gutiérrez, Concordance and homotopy. I. Fundamental

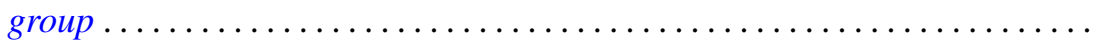

Richard I. Hartley, Metabelian representations of knot groups .............

Ted Hurley, Intersections of terms of polycentral series of free groups and free

Lie algebras ........................................

Roy Andrew Johnson, Some relationships between measures ............ 117

Oldřich Kowalski, On unitary automorphisms of solvable Lie algebras .......

Kee Yuen Lam, $K O$-equivalences and existence of nonsingular bilinear

maps...................................................

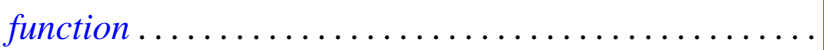

Robert A. Messer and Alden H. Wright, Embedding open 3-manifolds in compact 3-manifolds ............................

Gerald Ira Myerson, A combinatorial problem in finite fields. I . .

James Nelson, Jr. and Mohan S. Putcha, Word equations in a band of paths.

Baburao Govindrao Pachpatte and S. M. Singare, Discrete generalized Gronwall inequalities in three independent variables . .

William Lindall Paschke and Norberto Salinas, $C^{*}$-algebras associated with free products of groups ........................

Bruce Reznick, Banach spaces with polynomial norms ....

David Rusin, What is the probability that two elements of a finite group

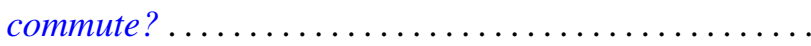

M. Shafii-Mousavi and Zbigniew Zielezny, On hypoelliptic differential operators of constant strength ...

Joseph Gail Stampfli, On selfadjoint derivation ranges .... . . .

Robert Charles Thompson, The case of equality in the matrix-valued triangle

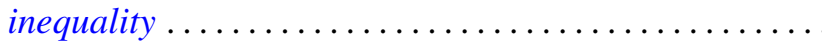

Marie Angela Vitulli, The obstruction of the formal moduli space in the negatively graded case. 